\title{
Primary Sjögren's Syndrome First Presenting as Hypokalemic Quadriparesis
}

\author{
Rolando MARTÍNEZ-GRANADOS, ${ }^{1}$ Guillermo DELGADO-GARCÍA, ${ }^{1}$ Martín WAH-SUÁREZ, ${ }^{1}$ \\ Nancy CONTRERAS-GARZA, ${ }^{1}$ Dionicio GALARZA-DELGADO ${ }^{1,2}$ \\ ${ }^{1}$ Department of Internal Medicine, University Hospital, Autonomous University of Nuevo León, Monterrey, Mexico \\ ${ }^{2}$ Division of Rheumatology, University Hospital, Autonomous University of Nuevo León, Monterrey, Mexico
}

\begin{abstract}
Hypokalemic paralysis and respiratory failure associated with distal renal tubular acidosis as first clinical manifestation of primary Sjögren's syndrome has been reported a few times. In this article, we describe the case of a previously healthy 38-year-old female patient who presented to the emergency department with a three-day history of dyspnea at rest and rapidly progressive generalized muscular weakness. Her initial work-up revealed an uncompensated hyperchloremic metabolic acidosis and severe hypokalemia. Additionally, urine $\mathrm{pH}$ of 8.0 and other findings consistent with distal renal tubular acidosis were found. We excluded the most common causes of distal renal tubular acidosis and finally established the diagnosis of primary Sjögren's syndrome. Our patient was treated with potassium citrate and later discharged on this treatment.

Keywords: Distal renal tubular acidosis; hyperchloremic metabolic acidosis; hypokalemic paralysis; respiratory insufficiency; Sjögren's syndrome.
\end{abstract}

Hypokalemic paralysis is a rare condition characterized by episodic weakness associated with hypokalemia. ${ }^{1}$ Several disorders have been associated with hypokalemic paralysis. However, most cases are due to renal tubular acidosis (RTA). ${ }^{2}$ RTA is suspected in the presence of hyperchloremic metabolic acidosis (HMA) and it is divided into three categories: proximal RTA, distal RTA (dRTA), and hyperkalemic RTA. dRTA is characterized by the inability to excrete the daily acid loads and by potassium imbalances. ${ }^{3}$ Primary Sjögren's syndrome (pSS) mainly affects exocrine glands; nevertheless it can impair renal function via tubular and glomerular injury. ${ }^{4}$ In adults, pSS has been identified as the leading cause of acquired dRTA. ${ }^{5}$ The relevance of this case stands in the atypical clinical presentation of symptomatic, severe hypokalemia as the first manifestation of pSS.

\section{CASE REPORT}

A 38-year-old female patient presented to the emergency department with a three-day history of dyspnea and progressive quadriparesis. This weakness progressed over a day until she was unable to stand up. She also reported fatigue and occasional acroparesthesias. On physical examination, she was alert and reactive, but unable to move any limb; she also was tachypneic and incapable to talk. Her blood pressure was $120 / 80 \mathrm{mmHg}$ and her oxygen saturation was $97 \%$ at room air. Chest and abdominal examinations were noncontributory. Deep tendon reflexes were absent and strength was $0 / 5$ in all four limbs, according to Medical Research Council scale. No Babinski response was found.

Level of serum potassium was $1.9 \mathrm{mmol} / \mathrm{L}$, sodium was $138 \mathrm{mmol} / \mathrm{L}$, chloride was $116.1 \mathrm{mmol} / \mathrm{L}$, total serum calcium was $8.6 \mathrm{mg} / \mathrm{dL}$, 
and serum albumin was $4.2 \mathrm{~g} / \mathrm{dL}$. The arterial blood gas analysis revealed a $\mathrm{pH}$ of 7.12 , partial pressure of carbon dioxide of $37 \mathrm{mmHg}$, and bicarbonate of $12.0 \mathrm{mEq} / \mathrm{L}$. Her erythrocyte sedimentation rate was $12 \mathrm{~mm} /$ hour. Urinalysis showed a $\mathrm{pH}$ of 8.0 without proteinuria, leukocyturia, or other alteration. Urinary sodium was $103 \mathrm{mmol} / \mathrm{L}$, urinary potassium was $30 \mathrm{mmol} / \mathrm{L}$, and urinary chloride was $57.3 \mathrm{mmol} / \mathrm{L}$. Her urine potassiumcreatinine ratio was 4.1 and her fractional excretion of potassium was $20 \%$. She also had a urinary anion gap of $76 \mathrm{mmol} / \mathrm{L}$ and a 24-hour urinary calcium of $449.8 \mathrm{mmol}$. Renal ultrasound revealed bilateral nephrocalcinosis. The rest of the laboratory tests were within the normal range (e.g., serum creatinine, liver function tests, thyroid function tests, enzyme-linked immunosorbent assay for human immunodeficiency virus, hepatitis B surface antigen, and anti-hepatitis $C$ virus).

We established the diagnosis of dRTA. A central venous catheter was then placed and she was started on intravenous potassium chloride and sodium bicarbonate. She clinically improved and her acid-base balance was restored. The patient was later switched to oral potassium citrate and remained solely on it. The most common causes of dRTA were rationally excluded. The remaining etiologies were autoimmune diseases, including systemic erythematous lupus, rheumatoid arthritis, and pSS. There was no history of fever, joint pain, skin rash, photosensibility, xerostomy, or parotid swelling. Her Schirmer's test was normal but she was positive for antinuclear antibodies (1:5120, SS A/Ro pattern). Thereafter, we tested her for specific anti-SS B/La and anti-SS A/Ro, and she was also positive (19 and $200 \mathrm{U} / \mathrm{mL}$, respectively). Due to the lack of typical presentation, we performed a salivary gland biopsy and thus diagnosed pSS according to the American College of Rheumatology/Sjögren's International Collaborative Clinical Alliance criteria. The patient was later discharged on potassium citrate and hydroxychloroquine. Before reporting her case, a written informed consent was obtained from the patient.

\section{DISCUSSION}

Hypokalemic paralysis is a rare disorder characterized by potentially fatal episodes of muscle weakness associated with hypokalemia, which can affect the respiratory muscles. ${ }^{2}$ Hypokalemic paralyses are divided into primary and secondary forms. Secondary forms are more common and are the result of acquired hypokalemia such as thyrotoxicosis, hyperaldosteronism, diabetic ketoacidosis, diarrhea, vomiting, drugs or RTA. ${ }^{6}$

The term RTA is applied to a group of defects in bicarbonate reabsorption, hydrogen ion excretion, or both. It is distinguished by a relatively normal glomerular filtration rate and a HMA. dRTA is characterized by impaired hydrogen ion secretion in the distal part of the nephron; this defect, in its severe form, leads to an inability to excrete the daily acid load, resulting in progressive hydrogen ion retention and a HMA. ${ }^{7}$ Although diminished $\mathrm{H}+$-ATPase activity is probably the most common defect in dRTA, impaired hydrogen ion secretion may be caused by several defects. ${ }^{8}$ dRTA is commonly associated with hypokalemia due to renal wasting. ${ }^{6}$ Occasionally, this hypokalemia is sufficiently severe to produce paralysis or respiratory arrest. ${ }^{9}$ In adults, autoimmune diseases are major causes of $\mathrm{dRTA}^{3}$ and this may be its presenting manifestation. Thus, adults with apparently idiopathic dRTA should be evaluated for these conditions. ${ }^{8}$

Distal RTA occurs in up to 5\% of patients with pSS; however, the prevalence of symptomatic dRTA was not assessed in this study. The first case secondary to pSS was reported by Shern and $\mathrm{Tu}^{10}$ in 1965. The mechanism by which pSS leads to dRTA is incompletely understood. ${ }^{11}$ One explanation has been found in immunocytochemical analyses of renal biopsies which have shown complete absence of H-ATPase pumps in the intercalated cell of the collecting tubules which is largely responsible for proton secretion. ${ }^{12}$ Another possible mechanism is the presence of high titers of an autoantibody directed against carbonic anhydrase II. Inhibition of this enzyme would be expected to impair intracellular generation of hydrogen ions. ${ }^{13}$

Sjögren's syndrome is among the most frequent systemic autoimmune diseases. Its main clinical features are ocular and oral dryness. ${ }^{14}$ Systemic complications can occur in pSS, and approximately $10 \%$ of patients have renal involvement. ${ }^{4}$ Some studies correlated RTA and high anti-SS A/Ro and anti-SS B/La 
level titers as a surrogate of pSS progression..$^{15,16}$ Additionally, other studies have suggested that lymphoplasmacytic infiltrates surrounding renal tubules are associated with renal tubular defects. ${ }^{17}$

It is recommended to treat dRTA with potassium citrate, which is an effective treatment for both the symptoms and complications of dRTA by restoring acid-base balance. It is unknown whether treatment with steroids has a positive effect on autoimmune dRTA. ${ }^{8}$

The clinical presentation of hypokalemic quadriparesis with respiratory failure as the first manifestation of pSS is very atypical. In this article, we tried to emphasize that hypokalemia can precipitate severe muscular weakness, and also that further evaluations are usually needed in order to elucidate the etiology of HMA.

\section{Declaration of conflicting interests}

The authors declared no conflicts of interest with respect to the authorship and/or publication of this article.

\section{Funding}

The authors received no financial support for the research and/or authorship of this article.

\section{REFERENCES}

1. Jandhyala SN, Madireddi J, Belle J, Rau NR, Shetty R. Hypokalaemic Periodic Paralysis- A Prospective Study of the Underlying Etiologies. J Clin Diagn Res 2015;9:17-9.

2. Ahlawat SK, Sachdev A. Hypokalaemic paralysis. Postgrad Med J 1999;75:193-7.

3. Rodríguez Soriano J. Renal tubular acidosis: the clinical entity. J Am Soc Nephrol 2002;13:2160-70.

4. Evans R, Zdebik A, Ciurtin C, Walsh SB. Renal involvement in primary Sjögren's syndrome. Rheumatology (Oxford) 2015;54:1541-8.

5. Duffles Amarante GB, Zotin MC, Rocha E, Delgado AG, Leite M Jr, Gomes CP. Renal tubular dysfunction in patients with primary Sjögren syndrome. Clin Nephrol 2014;81:185-91.

6. Fraer M. A mask and many faces: hypokalemic periodic paralysis. South Med J 2008;101:887.

7. Bagga A, Sinha A. Evaluation of renal tubular acidosis. Indian J Pediatr 2007;74:679-86.

8. Sengul E, Bunul F, Yazici A, Sengul A, Dindar S, Halhalli GS, et al. An unusual initial presentation of Sjögren's syndrome: severe hypokalemic paralysis secondary to distal renal tubular acidosis. Eurasian $\mathrm{J}$ Med 2013;45:218-21.

9. Yllmaz H, Kaya M, Özbek M, ÜUreten K, Safa Ylldırım İ. Hypokalemic periodic paralysis in Sjogren's syndrome secondary to distal renal tubular acidosis. Rheumatol Int 2013;33:1879-82.

10. Shearn MA, Tu WH. Nephrogenic diabetic insipidus and other defects of renal tubular function in sjoergren's syndrome. Am J Med 1965;39:312-8.

11. Both T, Zietse R, Hoorn EJ, van Hagen PM, Dalm VA, van Laar JA, et al. Everything you need to know about distal renal tubular acidosis in autoimmune disease. Rheumatol Int 2014;34:1037-45.

12. Cohen EP, Bastani B, Cohen MR, Kolner S, Hemken $\mathrm{P}$, Gluck SL. Absence of $\mathrm{H}(+)$-ATPase in cortical collecting tubules of a patient with Sjogren's syndrome and distal renal tubular acidosis. J Am Soc Nephrol 1992;3:264-71.

13. Takemoto F, Hoshino J, Sawa N, Tamura Y, Tagami $\mathrm{T}$, Yokota $\mathrm{M}$, et al. Autoantibodies against carbonic anhydrase II are increased in renal tubular acidosis associated with Sjogren syndrome. Am J Med 2005;118:181-4.

14. Cornec D, Jamin C, Pers JO. Sjögren's syndrome: where do we stand, and where shall we go? $\mathrm{J}$ Autoimmun 2014;51:109-14.

15. Both T, Hoorn EJ, Zietse R, van Laar JA, Dalm VA, Brkic Z, et al. Prevalence of distal renal tubular acidosis in primary Sjögren's syndrome. Rheumatology (Oxford) 2015;54:933-9.

16. Bossini N, Savoldi S, Franceschini F, Mombelloni S, Baronio M, Cavazzana I, et al. Clinical and morphological features of kidney involvement in primary Sjögren's syndrome. Nephrol Dial Transplant 2001;16:2328-36.

17. Dormohammadi Toosi T, Naderi N, Movassaghi S, Seradj MH, Khalvat A, Shahbazi F. Secondary Sjogren's syndrome presenting with hypokalemic periodic paralysis. Oxf Med Case Reports 2014;2014:135-7. 